\title{
50 Jahre Manuelle Medizin SAMM
}

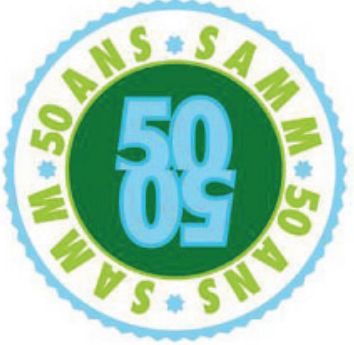

Sven Bradke

Dr. rer. publ. HSG, Geschäftsführer SAMM

* Die nächsten SAMM-Weiterbildungen für Einsteiger beginnen im Januar 2010 . Informationen hierzu finden Sie unter www.samm.ch

1 Binder A. Neck pain. BMJ Clin Evid. 2006;15:1-3.

2 Gross AR, Hoving JL, Haines TA, Goldsmith CH, Kay T, Aker P et al. A Cochrane review of manipulation and mobilization for mechanical neck disorders. Spine. 2004; 29(14):1541-8.

3 Milley M. et al. Does Cervical Manipulative Therapy Cause Vertebral Artery Dissection and Stroke? The Neurologist. 2008;14:66-73.

4 Thiel et al. Safety of Chiropractic Manipulation of the Cervical Spine.

A Prospective National Survey. Spine. 2007;32:2375-8.

Korrespondenz:

Schweizerische Ärztegesellschaft für Manuelle Medizin (SAMM) Röschstrasse 18

CH-9006 St.Gallen

Tel. 0712465181

info@samm.ch

www.samm.ch
Am 13. Dezember 1959 gründeten zwölf Ärzte die «Schweizerische ärztliche Arbeitsgemeinschaft für manipulative Therapie». Aus dieser Vereinigung ging später die «Schweizerische Ärztegesellschaft für manuelle Medizin SAMM» hervor. Dieser Standesorganisation gehören heute über 1300 Ärztinnen und Ärzte aus allen Sprachregionen der Schweiz an. Sie verfügen über eine spezielle Weiterbildung in Manueller Medizin sowie über einen entsprechenden FMH-Fähigkeitsausweis. Dieser wird aufgrund der geleisteten Fortbildungen alle fünf Jahre rezertifiziert. Die Gesellschaft sorgt im Auftrage der FMH für eine qualitativ hochstehende Weiter- und Fortbildung. Anlässlich des diesjährigen Jubiläums wurde ein neues deutschsprachiges Standardlehrbuch erarbeitet, das alle manual-medizinischen Handgriffe und Eingriffe in vierfarbigen Bildstrecken wiedergibt.

\section{Was ist Manuelle Medizin?}

Unter Manueller Medizin verstehen wir alle manuellen Einwirkungen auf den Patienten mit diagnostischer, therapeutischer und präventiver Zielsetzung. Die Manuelle Medizin kommt vor allem bei akuten und chronischen Beschwerden des Rückens beziehungsweise des Bewegungsapparates zum Einsatz. Zur Lösung diagnostizierter Funktionsstörungen werden beispielsweise Mobilisationen ohne Impuls, Manipulationen sowie verschiedene neuromuskuläre Inhibitionstechniken an Muskulatur und Weichteilen angewendet. Im SAMM-Leitbild steht deshalb der Leitsatz, dass wir die führende Ärztegesellschaft zur Behandlung von Funktionsstörungen des Bewegungsapparates (Rücken-, Gelenks- und Muskelbeschwerden) sein wollen.

\section{Wer betreibt Manuelle Medizin?}

Die Manuelle Medizin ist nicht nur für Spitalärzte, sondern insbesondere für Praxisärzte der Allgemeinmedizin, der Inneren Medizin sowie der Rheumatologie eine interessante und begehrte Zusatzausbildung. Als wissenschaftlich anerkannte medizinische Heilmethode erfüllt sie selbstredend auch die gesetzlich geforderten WZW-Kriterien. Deshalb wird sie auch über eine eigene Tarifposition im TARMED abgegolten.

\section{Hilft Manuelle Medizin?}

Die Wirksamkeit der Manuellen Medizin kann heute als evidenzbasiert bezeichnet werden, wie neuere Daten und Übersichten zeigen. Besonders gut dokumentiert sind Behandlungen der Halswirbelsäule [1,
2] und von akuten Rückenschmerzen, während die vor wenigen Jahren kontrovers geführte Diskussion über Komplikationen an der Halswirbelsäule sich wesentlich zugunsten der Manipulation beruhigt hat; es gibt lediglich ein sehr kleines Risiko bei unter 45-jährigen Patienten mit Nackenbeschwerden bei vorbestehender nicht-diagnostizierter bzw. verkannter spontaner Dissektionserkrankung der A. vertebralis [3, 4].

\section{Wie werde ich Manualmediziner?}

Einen manual-medizinischen Fähigkeitsausweis erhält, wer eine schweizerische Facharztausbildung mit FMH-Titel abgeschlossen, die gesamte SAMM-Weiterbildung berufsbegleitend besucht und nach zwei Jahren die praktische Prüfung bestanden hat.* Die Weiterbildung basiert auf 30 Unterrichtstagen mit klinischen Demonstrationen sowie auf einem entsprechenden Selbststudium im Umfang von rund 60 Stunden.

\section{Intensivierung der Zusammenarbeit}

In den nächsten Jahren möchte die SAMM das Fachgebiet und das Curriculum der Manuellen Medizin weiterentwickeln und wissenschaftlich vertiefen. Hierfür wird die Zusammenarbeit mit den verschiedenen europäischen Partnern sowie mit bestimmten Fakultäten noch gezielter verstärkt. Ziel ist es, die Gesellschaft wie im Ausland - universitär anzubinden. Vorerst feiern wir aber unser 50-Jahr Jubiläum. Unser Dank gilt dabei all unseren Vorfahren und Lehrmeistern, die sich über Jahre hinweg mit viel Engagement für unser schönes Fach und unser wunderbares medizinisches «Handwerk» eingesetzt haben.

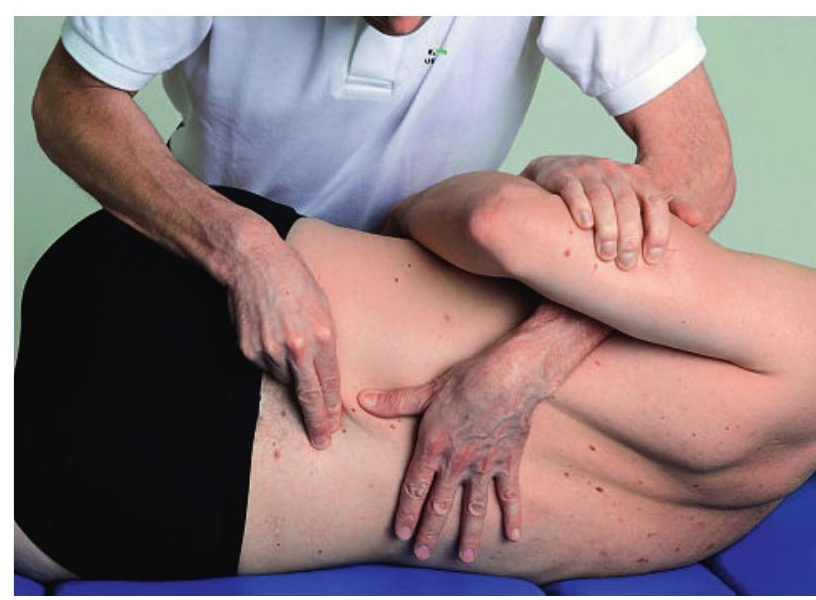

Die Wirksamkeit der Manuellen Medizin kann heute als evidenzbasiert bezeichnet werden. 